\title{
Iron concentrations in atherosclerotic plaque and serum in patients with carotid atherosclerosis
}

\author{
NM Tasic ${ }^{1}$, D Tasic ${ }^{1}$, M Veselinovic ${ }^{2}$, V Jakovljevic ${ }^{3}$, D Djuric ${ }^{4}$, D Radak ${ }^{1}$ \\ ${ }^{1}$ Dedinje Cardiovascular Institute, Faculty of Medicine, University of Belgrade, Belgrade, Serbia \\ ${ }^{2}$ Department of Rheumatology, Internal Clinic, CC »Kragujevac«, Faculty of Medical Sciences, \\ University of Kragujevac, Kragujevac, Serbia \\ ${ }^{3}$ Department of Physiology, Faculty of Medical Sciences, University of Kragujevac, Kragujevac, Serbia \\ ${ }^{4}$ Institute of Physiology "Richard Burian", Faculty of Medicine, University of Belgrade, Belgrade, Serbia
}

Received: February 4, 2015

Accepted after revision: February 25, 2015

\begin{abstract}
The aim of this study was to investigate the iron concentrations in serum and carotid plaque in patients with different morphology of carotid atherosclerotic plaque and compared with other metal ions. Carotid endarterectomy due to the significant atherosclerotic stenosis was performed in 91 patients. Control group consisted of 27 patients, without carotid atherosclerosis. Atherosclerotic plaques were divided into four morphological groups, according to ultrasonic and intraoperative characteristics. Iron, copper and zinc concentration in plaque, carotid artery and serum were measured by spectrophotometry. Serum iron concentrations were higher in patients with hemorrhagic plaques in comparison to the control group $(4.7 \mu \mathrm{mol} / 1 \pm 1.2$ vs. $2.1 \mu \mathrm{mol} / 1 \pm 0.8, p<0.05)$. Iron concentrations were higher in patients with hemorrhagic plaques in comparison to fibrolipid plaques $(72.1 \pm 14.3 \mu \mathrm{g} / \mathrm{g}$ vs. $39.3 \pm 22.9 \mu \mathrm{g} / \mathrm{g}$; $p<0.05)$. Negative significant correlation was found for zinc in serum and plaque iron concentration in patients $(p<0.05)$. We also demonstrated positive significant correlation for copper and iron in serum $(p<0.05)$. The data obtained in the current study are consistent with the hypothesis that high iron levels may contribute to atherosclerosis and its complications as factors in a multifactorial disease.
\end{abstract}

Keywords: carotid plaque, atherosclerosis, iron, zinc, copper

Over the last 30 years, several studies in animals and humans assessed the effect of increased body iron levels on atherosclerosis, yielding conflicting results.

Iron is a key element in many biochemical processes and shortage of iron causes damage to cells and organs. On the other hand, excess iron could be harmful because it is able to catalyse the formation of highly reactive oxygen and hydrogen radicals when present in the unbound state. Transition metal ions, established catalysts of protein, lipid and DNA oxidation, are present at elevated levels in human atherosclerotic plaques, and in some animal models, consistent with the hypothesis that metal ions contribute to both plaque formation and its destabilization $(19,21,23)$, though the role of metal ions remains controversial $(16,29)$.

Epidemiological studies have reported an increased incidence of cardiovascular disease (CVD) in subjects with overt iron overload $(7,8,19)$. Recent studies on cardiovascular evaluation and outcome in high- versus low-frequency blood donors have demonstrated improvements in surrogate markers of vascular health such as decreased oxidative stress, and

\footnotetext{
Corresponding author: Nebojsa M. Tasic, MD

"Dr Aleksandar D. Popovic" Cardiovascular Research Center, Dedinje Cardiovascular Institute Milana Tepića 1, 11040 Belgrade, Serbia \& Montenegro

Phone: +(381-11) 3601669; Fax: +(381-11) 2666445; E-mail: nebtasa@yahoo.com
} 
enhanced vascular reactivity (3). Several population studies have reported a direct association between increased iron intake, body iron stores, and CVD risk; in particular, elevated intake of heme iron is associated with increased CVD events, and increased total body iron stores with myocardial infarction (MI) (24).

Although convincing evidence has been previously provided for elevated levels of iron and copper ions in advanced human lesions $(21,23)$, and for a correlation between metal ion levels and the extent of protein oxidation (23), these studies have not examined specific populations. Current evidence and pathophysiological considerations suggest that the increased intraplaque iron deposition may be associated with increased oxidative stress, affecting the stability of the carotid plaque (12). Thus, data on the abundance of metal ions in atherosclerotic lesions, and its potential correlation with clinical symptoms is lacking. A number of studies have reported associations between metals and atherosclerosis, evaluated by carotid artery intima-media thickness (IMT) measurement (11).

The present study was aimed at examining the relationship between measures of iron status in serum and carotid plaque in patients with different morphology of carotid atherosclerotic plaque. We compared the concentrations of iron with concentrations of metal ions (zinc, copper) in serum and carotid plaque in patients.

\section{Patients and Methods}

\section{Patients}

The study group consisted of 118 subsequent patients (mean age $63 \pm 8$ yrs; $54.2 \%$ males) admitted to the Department of Vascular Surgery at Dedinje Cardiovascular Institute for carotid surgery. Carotid endarterectomy due to the significant atherosclerotic stenosis and symptoms of cerebrovascular insufficiency was performed in 91 patients (mean age $64.2 \pm$ $6.9 \mathrm{yrs}, 62.6 \%$ males). Control group consisted of 27 patients (mean age $58.4 \pm 9.3 \mathrm{yrs}$, $25.9 \%$ males) without carotid atherosclerosis which were operated due to the symptomatic kinking and coiling of carotid artery.

All patients underwent neurological, cardiological and vascular surgeon exam, carotid artery ultrasound measurements and were treated surgically. Blood samples were drawn from all patients before surgery and sera were analyzed for the concentrations of iron, copper and zinc.

Tissue samples, atherosclerotic plaques from atherosclerotic patients and normal carotid tissue from the control group patients were analyzed for the concentrations of iron, copper and zinc. In atherosclerotic subjects, atherosclerotic plaques were divided into four morphological groups, that is fibrolipid, hemorrhagic, ulcerated and calcified plaque, according to ultrasonic and intraoperative characteristics (4).

The study was approved by the Ethics Committee of the Dedinje Cardiovascular Institute. Written consent was obtained from all patients before they entered the study.

\section{Analytical methods}

Copper and zinc concentrations in plaque, carotid artery tissue and serum were estimated by means of flame atomic absorption spectrophotometry (AAS) using the Varian AA-5 instrument and the instrumental conditions recommended by the producer (acetylene/air flame, wavelenght: $\lambda \mathrm{Cu}=324.75 \mathrm{~nm}, \lambda \mathrm{Zn}=213.86 \mathrm{~nm} ; \lambda \mathrm{Fe}=248.3 \mathrm{~nm}$ ). 


\section{Statistical analysis}

Data are presented graphically as box-plots, showing the median, minimum and maximum value. Differences in quantitative variables were assessed using the Mann-Whitney U test. The associations between groups were analyzed by Spearman's correlation. A $p$ value $<0.05$ was considered statistically significant. Data were analyzed by SPSS version 10 (SPSS Inc, Chicago, Illinois, USA).

\section{Results}

Our patient cohort exhibited most of the classic atherosclerosis risk factors (Table I). The percentage of hypertensive, smoking subjects was higher in patients with carotid plaque than in controls $(p<0.05)$. The presence of diabetes showed statistically significant differences between patients and controls $(p<0.05)$.

Table I. Baseline characteristics of study sample

\begin{tabular}{|c|c|c|c|}
\hline Variable & $\begin{array}{l}\text { Atherosclerotic subjects } \\
\qquad N=91\end{array}$ & $\begin{array}{c}\text { Control subjects } \\
\qquad N=27\end{array}$ & $p$ values \\
\hline Age & $64.2 \pm 6.9$ & $58.4 \pm 9.3$ & $p>0.05$ \\
\hline Hypertension (\%) & 90.1 & 59.3 & $p<0.05$ \\
\hline Smokers $(\%)$ & 67 & 40.7 & $p<0.05$ \\
\hline Diabetes (\%) & 26.4 & 14.8 & $p<0.05$ \\
\hline Family history (\%) & 49.4 & 55.6 & $p>0.05$ \\
\hline Carotid artery bruit (\%) & 90.1 & 33.3 & $p<0.05$ \\
\hline
\end{tabular}

Atherosclerotic subjects were divided in four groups according to different morphology of analyzed atherosclerotic plaques (4). First (I) group (22; 18.6\%) represents patients with fibro-lipid atherosclerotic plaque.

In the second (II) group there were the patients with hemorrhagic plaque ( 8 patients; $6.8 \%$ ), in the third (III) group those with ulcerated plaque (30 patients; $25.4 \%$ ) and the fourth (IV) group consisted of patients with calcified atherosclerotic plaque (30 patients; $25.4 \%$ ). Patients of the control group, without significant atherosclerotic changes in the carotid artery constitute the fifth group (27 patients; $22.9 \%$ ).

Distribution of iron concentrations in plaque, carotid tissue and serum are shown in Figs 1 and 2 , respectively.

Our study revealed significantly higher average serum iron concentration in patients with hemorrhagic plaque (II) compared to patients in the control group ( $4.7 \mu \mathrm{g} / 1 \pm 1.2 \mathrm{vs} .2 .1$ $\mu \mathrm{g} / 1 \pm 0.8, p<0.05)$. The average values of iron concentrations in different morphology atherosclerotic plaques and normal carotid tissue were similar (Fig. 1).

We found significantly higher average iron plaque concentration in patients with hemorrhagic plaque (II) in comparison to patients with fibro-lipid (I) plaques $(72.1 \pm 14.3$ $\mu \mathrm{g} / \mathrm{g}$ vs. $39.3 \pm 22.9 \mu \mathrm{g} / \mathrm{g} ; p<0.05$ ) (Fig. 2 ).

We also demonstrated negative significant correlation for iron plaque concentration and zinc in serum in atherosclerotic patients $(p=0.042)$ (Fig. 3).

Positive and significant correlation coefficients were calculated for iron in serum and copper in serum in atherosclerotic patients $(p=0.017)$ (Fig. 4). 

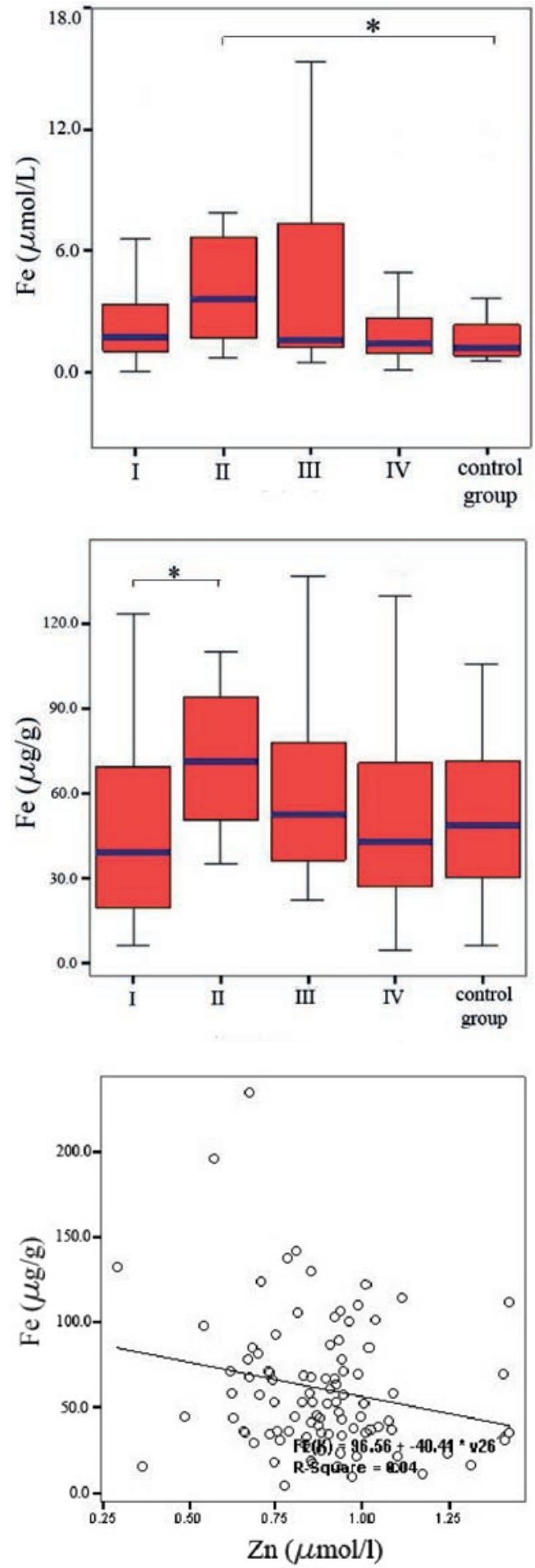

Fig. 1. Average serum iron (Fe) concentration in different morphological groups of atherosclerotic plaques. Groups of patients: I - with fibro-lipid atherosclerotic plaque; II - with hemorrhagic plaque;

III - with ulcerated atherosclerotic plaque;

IV - with calcified atherosclerotic plaque.

$* p<0.05$ (statistically significant difference between the groups II and control group)

Fig. 2. Average plaque iron (Fe) concentration in different morphological groups of atherosclerotic plaques. Groups of patients: I - with fibro-lipid atherosclerotic plaque; II - with hemorrhagic plaque;

III - with ulcerated atherosclerotic plaque;

IV - with calcified atherosclerotic plaque.

$* p<0.05$ (statistically significant difference between the groups I and II)

Fig. 3. Analysis of the association of iron (Fe) in the carotid plaque and zinc $(\mathrm{Zn})$ in serum of the patients studied.

$p=0.042 ; \mathrm{FE}(\mathrm{K}) ;$ plaque iron $(\mathrm{Fe})$ concentration 


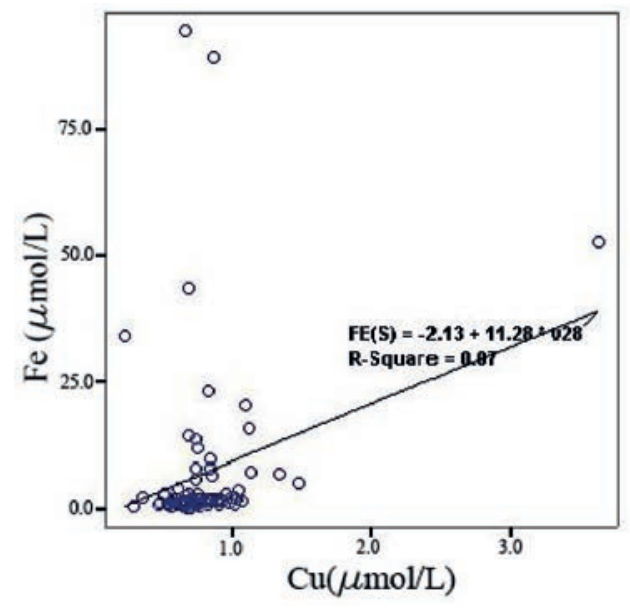

Fig. 4. Analysis of iron (Fe) concentrations in serum and copper $(\mathrm{Cu})$ in the serum of patients with pronounced atherosclerosis.

$p=0.017 ; \mathrm{FE}(\mathrm{S}) ;$ iron concentrations in serum

Distribution of patients according to gender in relation to the value of iron in carotid plaque showed higher values of iron in groups of patients with pronounced atherosclerosis in men than women $(p<0.05)$ (data not showed).

\section{Discussion}

This study complements previous studies on the levels of metal ions in advanced human atherosclerotic lesions $(20,21,23)$. Unlike these previous studies this work has quantified these parameters in two sample groups. It was hypothesised that lesions from patients with carotid plaques would contain higher levels of metal ions. The data obtained from these groups were compared to control subjects without significant atherosclerotic changes in carotid artery. Previous studies have reported that advanced atherosclerotic lesions have higher metal ion levels than initial lesions or healthy tissue $(11,21,22)$.

Site-specific effects on the development of atherosclerosis are well established, with a recent review (5) emphasizing the importance of studying the dynamics and location of trace metal ions in vivo, in order to assess the significance of these elements in disease development. Iron is present in the atherosclerotic plaque at concentrations that appreciably exceed those found in healthy arterial tissue (24). This iron is not simply an inert component of the plaque. Some of the arterial iron is redox active and the ferritin- and hemosiderin-bound iron compartment remains a reservoir of potentially reactive iron within lesions. We have shown significantly higher iron plaque concentration in patients with hemorrhagic plaque in comparison to patients with fibro-lipid plaques. The findings support the concept that arterial plaque iron is a modifiable risk factor for atherogenesis (24). The finding that human atherosclerotic plaques contain redox-active iron that could promote free radical formation and lipid peroxidation, further suggested a role for iron in atherosclerosis that may be eventually responsible for progressive oxidative damage in atherosclerotic lesions. Recent studies show that iron may play a key role in plaque vulnerability and contributes to plaque hemorrhage $(13,14)$. Biologically plausible mechanisms for this association have been described, however, epidemiological studies on iron status and CVD risk have provided conflicting result (9). Extreme conditions of iron deficiency, as well as of iron overload, are 
associated with modestly increased CVD risk, although with different proposed mechanisms (9). The findings support the concept that arterial plaque iron is a modifiable risk factor for atherogenesis. Stadler et al. (21) quantified iron in ex vivo carotid lesions and in healthy human arteries. They found elevated levels of iron in the intima of lesions compared with healthy controls. In a later work (23), iron levels in human atherosclerotic plaque correlated positively and strongly with multiple markers of protein oxidation.

Our study revealed significantly higher average serum iron concentration in patients with hemorrhagic plaque compared to patients in the control group. The results of several human studies strongly suggested a relationship between body iron levels and atherosclerosis. According to the epidemiological studies, high systemic iron levels, monitored by serum ferritin levels or transferrin saturation, positively correlated with an increased risk of myocardial infarction $(7,18)$. A protective effect of iron depletion that may have multiple beneficial consequences is decreased availability of redox-active iron in vivo (25). It has been proposed that the amount of free iron available at sites of oxidative or inflammatory injury is a function of the stored iron level and that the availability of redox-active iron in vivo approaches its minimum in the state of iron depletion. There is significant experimental support for this concept (24). In particular, it has been shown that removal of stored iron from the body can decrease the amount of iron deposition within atherosclerotic lesions in animal studies. Depletion of lesion iron levels in vivo by phlebotomy, systemic iron chelation treatment or dietary iron restriction reduces lesion size in these studies and increases plaque stability $(10,15,27)$. The original formulation of the iron hypothesis did not specify a mechanism. There may well be multiple mechanisms by which iron depletion protects against atherosclerosis. Paramonov et al. did not register significant difference in serum iron in patients with different forms of coronary heart disease and healthy control subjects (17).

Distribution of patients according to gender in relation to the value of iron in carotid plaque showed higher values of iron in groups of patients with pronounced atherosclerosis in men than women. Wolff et al. examined the correlation of serum ferritin and IMT thickness in the general population. Analysis of the results shows a significant positive correlation between these parameters only in men. In women ferritin had no predictive value for carotid atherosclerosis (28). This suggests that the observed difference between men and women may be a result of physiological differences (i.e. menstruation and hormone secretion) that affect iron storage.

We also demonstrated negative significant correlation for iron plaque concentration and zinc in serum. Studies have reported that low zinc concentration may have an important role in the pathogenesis of atherosclerosis (2). Low serum zinc/24-hour urine zinc ratio is associated with angiographically severe atherosclerosis. It was observed that low zinc levels were associated with patients with cardiovascular diseases $(1,6)$.

Epidemiological studies have sought to link various measures of iron and copper with the incidence of cardiovascular disease, after the suggestion that the development of disease is linked to iron stores, with iron-deficiency offering protection (21). These data are equivocal, with positive associations detected in some studies but not others. Positive and significant correlation coefficients were calculated for iron in serum and copper in serum in our patients. Iron and copper can promote oxidative damage to extracellular matrix components (21). These elevated levels of metal ions may therefore affect plaque stability and propensity to rupture.

Additional studies are needed to better characterize the significance of the various concentrations of iron within the plaque and to assess the clinical utility of non-invasive 
measures of plaque iron in the clinical determination of plaque stability. Despite the large number of studies having been published to date, the role of iron in CVD still generates a fair amount of debate (26).

However, the impact of iron on atherosclerosis is still debated. Comprehensive understanding of the role of iron and other metal ions on atherogenesis may lead to the development of improved diagnosis and treatment of atherosclerosis. Future studies are required to clearly address whether iron overload is a risk factor for atherosclerosis and what iron source - systemic or tissue iron - affects mainly the atherosclerotic process. Future studies are needed to clarify the real effect of iron on CVD.

\section{REFERENCES}

1. Afridi H, Kazi T, Kazi N, Kandhro G, Baig J, Jamali M, Arain M, Shah A: Interactions between cadmium and zinc in the biological samples of Pakistani smokers and nonsmokers cardiovascular disease patients. Biol. Trace Elem. Res. 139(3), 257-268 (2011)

2. Allen-Redpath K, Ou O, Beattie J, Kwun I, Feldmann J, Nixon G: Marginal dietary zinc deficiency in vivo induces vascular smooth muscle cell apoptosis in large arteries. Cardiovasc. Res. 99(3), 525-534 (2013)

3. Bagheri B, Shokrzadeh M, Mokhberi V, Azizi S, Khalilian A, Akbari N, Habibi V, Yousefnejad K, Tabiban S, Nabati M: Association between serum iron and the severity of coronary artery disease. I. Cardiovasc. Res. J. 7, 95-98 (2013)

4. Beletsky V: Ultrasound Densitometric Analysis of carotid plaque composition pathoanatomic correlation. Stroke 27, 2173-2177 (1996)

5. Easter R, Qilin Chan, Lai B, Ritman E, Caruso J, Zhenyu Qin: Vascular metallomics: copper in the vasculature. Vasc. Med. 15, 61-69 (2010)

6. Giannoglou G, Konstantinou D, Kovatsi L, Chatzizisis Y, Mikhailidis D: Association of reduced zinc status with angiographically severe coronary atherosclerosis: a pilot study. Angiology 61(5), 449-455 (2010)

7. Holay M, Choudhary A, Suryawanshi S: Serum ferritin a novel risk factor in acute myocardial infarction. Indian. Heart J. 64, 173-177 (2012)

8. Kang P, Liu T, Tian C, Zhou Y, Jia C: Association of total iron binding capacity with coronary artery disease. Clin. Chim. Acta 413, 1424-1429 (2012)

9. Lapice E, Masulli M, Vaccaro O: Iron deficiency and cardiovascular disease: an updated review of the evidence. Curr. Atheroscler. Rep. 15(10), 358 (2013)

10. Lee H, Chiu L, Lee T, Tsai H, Chau L: Dietary iron restriction increases plaque stability in apolipoprotein-edeficient mice. J. Biomed. Sci. 30, 510-517 (2003)

11. Lind PM, Olsén L, Lind L: Circulating levels of metals are related to carotid atherosclerosis in elderly. Sci. Total Environ. 416, 80-88 (2012)

12. Lioupis C, Barbatis C, Drougou A, Koliaraki V, Mamalaki A, Klonaris C, Georgopoulos S, Andrikopoulos V, Bastounis E: Association of haptoglobin genotype and common cardiovascular risk factors with the amount of iron in atherosclerotic carotid plaques. Atherosclerosis 216(1), 131-138 (2013)

13. Mascitelli L, Goldstein M: Might intraplaque hemorrhage represents the proof-of-concept that iron plays a causal role in plaque vulnerability? J. Cardiovasc. Med. (Hagerstown) 15(12), 882-883 (2014)

14. Michel J, Delbosc S, Ho-Tin-Noé B, Leseche G, Nicoletti A, Meilhac O, Martin-Ventura J: From intraplaque haemorrhages to plaque vulnerability: biological consequences of intraplaque haemorrhages. J. Cardiovasc. Med. (Hagerstown) 13(10), 628-634 (2012)

15. Minqin R, Rajendran R, Pan N, Kwong-Huat T, Ong W, Watt F, Halliwell B: The iron chelator desferrioxamine inhibits atherosclerotic lesion development and decreases lesion iron concentrations in the cholesterol-fed rabbit. Free Radic. Biol. Med. 38, 1206-1211 (2005)

16. Muñoz-Bravo C, Gutiérrez-Bedmar M, Gómez-Aracena J, García-Rodríguez A, Navajas J: Iron: protector or risk factor for cardiovascular disease? Still controversial. Nutrients 5(7), 2384-2404 (2013)

17. Paramonov A, Moiseev S, Fomin V, Kopelëva M, Stankevich L, Martynov A, Mukhin N: Ferritin and other acute phase proteins in various forms of coronary heart disease. Klin. Med. (Mosk). 83(2), 25-29 (2005)

18. Rajapurkar M, Shah S, Lele S, Hegde U, Lensing S, Gohel K, Mukhopadhyay B, Gang S, Eigenbrodt M: Association of catalytic iron with cardiovascular disease. Am. J. Cardiol. 109, 438-442 (2011) 
19. Raman S, Winner M 3rd, Tran T, Velayutham M, Simonetti O, Baker P, Olesik J, McCarthy B, Ferketich A, Zweier J: In vivo atherosclerotic plaque characterization using magnetic susceptibility distinguishes symptomproducing plaques. JACC Cardiovasc. Imaging 1, 49-57 (2008)

20. Stadler N, Heeneman S, Vöö S, Stanley N, Giles G, Gang B, Croft K, Mori T, Vacata V, Daemen M, Waltenberger $\mathrm{J}$, Davies M: Reduced metal ion concentrations in atherosclerotic plaques from subjects with type 2 diabetes mellitus. Atherosclerosis 222(2), 512-518 (2012)

21. Stadler N, Lindner R, Davies M: Direct detection and quantification of transition metal ions in human atherosclerotic plaques: evidence for the presence of elevated levels of iron and copper. Arterioscler. Thromb. Vasc. Biol. 24, 949-954 (2004)

22. Stadler N, Stanley N, Heeneman S, Vacata V, Daemen M, Bannon P, Waltenberger J, Davies M: Accumulation of zinc in human atherosclerotic lesions correlates with calcium levels but does not protect against protein oxidation. Arterioscler. Thromb. Vasc. Biol. 28, 1024-1030 (2008)

23. Stanley N, Stadler N, Woods A, Bannon P, Davies M: Concentrations of iron correlate with the extent of protein, but not lipid, oxidation in advanced human atherosclerotic lesions. Free Radic. Biol. Med. 40, 1636-1643 (2006)

24. Sullivan J: Iron in arterial plaque: modifiable risk factor for atherosclerosis. Biochim. Biophys. Acta 1790, $718-723$ (2009)

25. Sullivan J: Is stored iron safe? J. Lab. Clin. Med. 144, 280-284 (2004)

26. Vinchi F, Muckenthaler M, Da Silva MC, Balla G, Balla J, Jeney V: Atherogenesis and iron: from epidemiology to cellular level. Front. Pharmacol. 5, 94 (2014)

27. Rajendran R, Minqin R, Ronald J, Rutt B, Halliwell B, Watt F: Does iron inhibit calcification during atherosclerosis? Free Radic. Biol. Med. 53(9), 1675-1679 (2012)

28. Wolff B, Völzke H, Lüdemann J, Robinson D, Vogelgesang D, Staudt A, Kessler C, Dahm J, John U, Felix S: Association between high serum ferritin levels and carotid atherosclerosis in the study of health in Pomerania (SHIP). Stroke 35, 453-457 (2004)

29. Yuan X, Li W: Iron involvement in multiple signaling pathways of atherosclerosis: a revisited hypothesis. Curr. Med. Chem. 15, 2157-2172 (2008) 